\title{
Variation Law Research of Finite Length Cylinder's RCS
}

\author{
Yehua Li ${ }^{*}$, Shan He, Daqing Huang \\ Beijing Institute of Aeronautical Material, Beijing, China
}

Email address:

rongchuanligang@163.com (Yehua Li), biamhs333@sina.com (Shan He), hadbiam@163.com (Daqing Huang)

${ }^{*}$ Corresponding author

\section{To cite this article:}

Yehua Li, Shan He, Daqing Huang. Variation Law Research of Finite Length Cylinder's RCS. Science Discovery. Vol. 4, No. 4, 2016, pp. 243-248. doi: 10.11648/j.sd.20160404.17

Received: May 16, 2016; Accepted: May 18, 2016; Published: July 20, 2016

Abstract: In order to analyse variation law research of finite length cylinders' RCS, this paper contrasts and analyses the calculation methods of RCS, designs different cylinders in size for calculating their RCS, and makes models of cylinders for measuring RCS. By analyzing the data of measure and calculation, FEKO software is verified having high accuracy in calculating RCS. This paper calculates cylinders' RCS in conditions of the incident electromagnetic wave perpendicular to circular surface and cylindrical surface,and calculates incidence angles for motivating surface travelling wave to acquire maximal value in orientation diagram. On the basis of calculation, this paper summarizes the influence of cylinders' diameters and lengths to RCS and the estimate angle of surface travelling wave's maximal value in orientation diagram. The conclusions can be referred in design and engineering application.

Keywords: Cylinder, RCS, FEKO, Surface Travelling Wave

\section{有限长圆柱体RCS变化规律研究}

\author{
李业华, 何山, 黄大庆 \\ 北京航空材料研究院, 北京, 中国

\section{邮箱} \\ rongchuanligang@163.com(李业华), biamhs333@sina.com(何山), hadbiam@163.com(黄大庆)
}

\begin{abstract}
摘要: 为分析有限长圆柱体雷达散射截面 (RCS) 的变化规律, 对比分析了RCS的计算方法, 使用FEKO软件设计不同尺 寸的圆柱体计算RCS, 制作实体模型测量RCS, 分析得出FEKO软件计算RCS具有很高的准确性。选择入射波垂直于圆面、 垂直于柱面、激起表面行波最大辐射方向的掠入射角度进行计算研究, 总结出圆柱体直径和长度对RCS变化规律的影响, 以及对表面行波最大辐射方向的角度估算准确性的影响, 得出的结论可作为设计和工程应用的参考。
\end{abstract}

关键词: 圆柱体, RCS, FEKO, 表面行波

\section{1. 引言}

圆柱体作为特殊的形状体在隐身设计中经常涉及，以 往的圆柱体RCS研究中多数探讨了无限长圆柱体的RCS计算 及变化情况。李有权等对无限长圆柱体在垂直极化下柱面 RCS的计算方法作了比较, 得出矩量法有较高的精度, 时域
有限差分法效率较高但在某些角度存在误差 $[1]$; 郝雷等研 究了无限长理想导电圆柱体爬行波后向RCS, 总结出电磁波 垂直于柱面入射时后向RCS随圆柱体尺寸增大而减小 [2]; 夏学仁等根据GTD-MOM技术提出了一种物理模式基概念, 求 解了有限细长圆柱体的RCS [3]; 郭华昌等应用SBR/FDTD混 合算法求解了圆柱形复杂终端腔体的RCS $[4]$ 。在实际应用 
中圆柱体都为有限长, 在瑞利区、谐振区、光学区目标体 的尺寸对RCS产生的影响不同, 圆柱体的直径及长度将影响 电磁波在表面的传播和衰减, 对有限长圆柱体RCS需要进一 步研究和探讨。本文讨论了RCS的计算方法, 使用FEKO软件 计算不同尺寸圆柱体的RCS，并制作实体模型测量RCS，通 过对比分析得出FEKO软件计算圆柱体RCS 具有很高的精度, 进而计算了多种直径及长度的有限长圆柱体RCS, 分析出入 射波分别垂直于圆面和柱面时在一定条件下圆柱体RCS随 直径、长度、入射频率的变化情况, 以及从圆面转到柱面 过程中表面行波方向图最大值出现的角度与经验公式计算 结果之间的偏差。本文首次对有限长圆柱体多角度的RCS 变化规律进行了计算研究, 本文得出的计算结果及变化规 律可为相关的研究工作和工程应用提供数据支持和参考。

\section{RCS计算方法}

目标体的电磁散射特性根据目标体尺寸 L与入射波长 $\lambda$ 的比值划分为瑞利区、谐振区、光学区三种类型。瑞利 区的雷达截面可用经验公式 (1) 表示 [5]:

$$
\sigma=\frac{\pi}{4} K^{4} V^{2} F^{2}
$$

式中: $K$ 一波数, $K=2 \pi / \lambda$;

V一目标体的体积;

$\mathrm{F}$ 一目标体形状的一个系数;

在瑞利区目标体的体积对RCS影响很大, 而外形对RCS 影响较小。在谐振区目标体尺寸与入射波波长处于同一数 量级, 物体各部分间的影响比较明显。在光学区目标体尺 寸远大于入射波波长，散射体的各个部分之间的相互作用 很小, 目标体的几何结构对总的散射场影响明显 [6-7]。

目标体的RCS求解方法有经典解法、积分方程解法、高 频解法。经典解法是根据波动方程和散射体的边界条件求得 的严格级数解, 这种方法只适用于一些简单的目标。高频方 法是假设目标体各部分之间相互作用很小将其忽略, 简化了 求解过程。高频解法主要有几何光学 (GO) 法、几何绕射理论 (GTD)、物理光学 $(\mathrm{PO})$ 法、物理绕射理论 $(\mathrm{PTD}) 、$ 、等效电磁流 法 (MEC) 等。高频方法虽然在计算时间和对计算机性能要求
上有一定的优势，但各种高频解法都是近似算法，应用区域 上都有局限性, 不能在整个目标体上实现准确的计算 [8]。

积分方程解法适用于任意形状的目标体, 通过求解 Stratton-Chu积分方程（2）、（3）可求得散射场 [9], 进而求解出雷达散射截面, 如式 (4) 所示。

$$
\begin{aligned}
& \vec{E}^{S}=\int_{V}\left(j \omega \mu \vec{J} \Psi-\vec{M} \times \nabla \Psi+\frac{1}{\varepsilon} \rho \nabla \Psi\right) d V+ \\
& \oint_{S}[j \omega \mu(\hat{n} \times \vec{H}) \Psi+(\hat{n} \times \vec{E}) \times \nabla \Psi+(\hat{n} \cdot \vec{E}) \nabla \Psi] d S \\
& \vec{H}^{S}=\int_{V}\left(j \omega \varepsilon \vec{M} \Psi+\vec{J} \times \nabla \Psi+\frac{1}{\mu} \rho^{*} \nabla \Psi\right) d V- \\
& \oint_{S}[j \omega \varepsilon(\hat{n} \times \vec{E}) \Psi+(\hat{n} \times \vec{H}) \times \nabla \Psi+(\hat{n} \cdot \vec{H}) \nabla \Psi] d S
\end{aligned}
$$

式中: $\Psi$ 一自由空间的格林函数, $\Psi=\frac{e^{j k R}}{4 \pi R}$

$$
\sigma=4 \pi \lim _{R \rightarrow \infty} R^{2} \frac{\left|E_{S}\right|^{2}}{\left|E_{\mathrm{i}}\right|^{2}}=4 \pi \lim _{R \rightarrow \infty} R^{2} \frac{\left|H_{S}\right|^{2}}{\left|H_{\mathrm{i}}\right|^{2}}
$$

矩量法是积分方程解法中代表性的方法, 矩量法将积 分方程化为矩阵方程, 通过矩阵求逆解出物体表面的感应 电流, 从而计算出散射场和雷达散射截面。FEKO软件以矩 量法 (MOM) 为基础, 集成了多层快速多极子方法 (MLFMA)、 物理光学法 (PO) 等多种算法。在FEKO软件中使用矩量法无 需用网格划分传播空间和设置边界吸收条件, 如计算机硬 件条件允许, 可求解任意结构的目标体RCS [10]。

\section{FEKO软件计算验证}

圆柱体具有对称性, 可对从垂直于圆面 $\left(0^{\circ}\right)$ 转到垂 直于柱面 $\left(90^{\circ}\right)$ 的RCS进行研究 [11]。设计三种规格的圆 柱体选择不同频率计算水平极化 (HH) 条件下的RCS, 并与 测量值对比如图1 图3所示。将 $0^{\circ} \sim 90^{\circ}$ 的RCS计算平均值 $\sigma_{\mathrm{c}}$ 与测量平均值 $\sigma_{\mathrm{m}}$ 对比如表 1 所示。图4为FEK0软件计算圆 柱体RCS的截图, 图5为圆柱体在暗室内的测量图片, 使用的 RCS测量系统在目标体RCS $\geqslant-25 \mathrm{dBm}^{2}$ 时测量不确定度 $\leqslant 1.0 \mathrm{~dB}$ 。

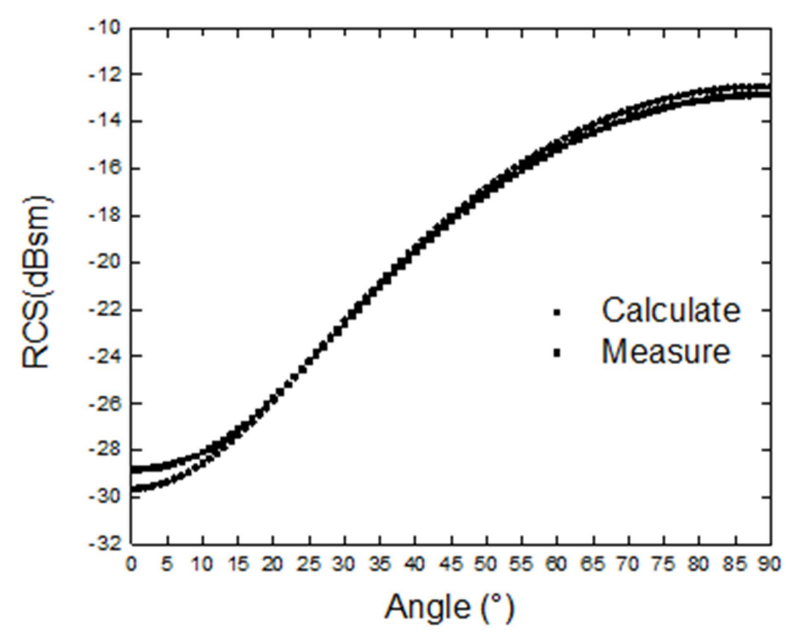

图1 直径 $50 \mathrm{~mm}$ 长度 $100 \mathrm{~mm}$ 的圆柱体在 $1 \mathrm{GHz}$ 的RCS。 


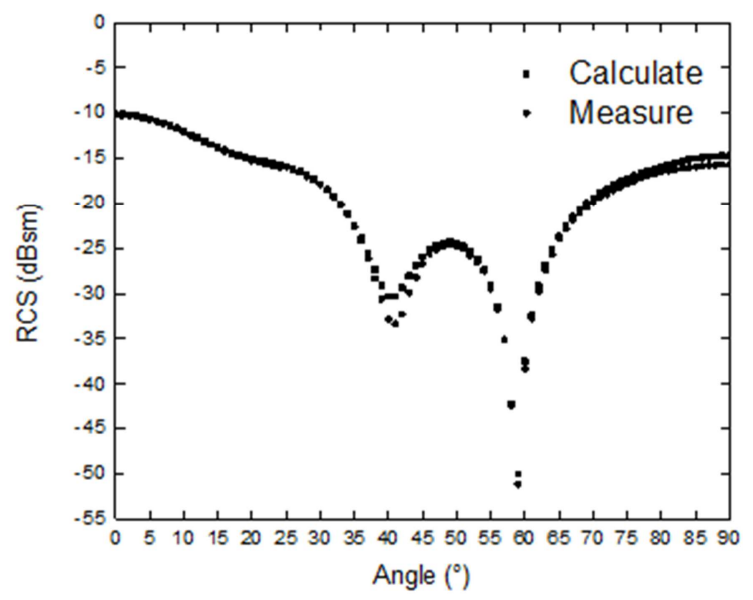

图2 直径 $100 \mathrm{~mm}$ 长度 $100 \mathrm{~mm}$ 的圆柱体在 $3 \mathrm{GHz}$ 的 $\mathrm{RCS}$ 。

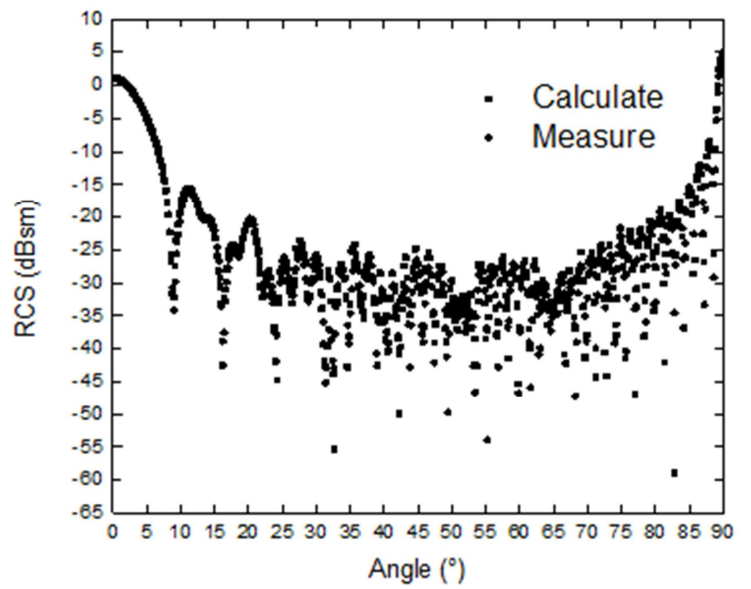

图3 直径 $100 \mathrm{~mm}$ 长度 $500 \mathrm{~mm}$ 的圆柱体在 $12 \mathrm{GHz}$ 的 RCS。

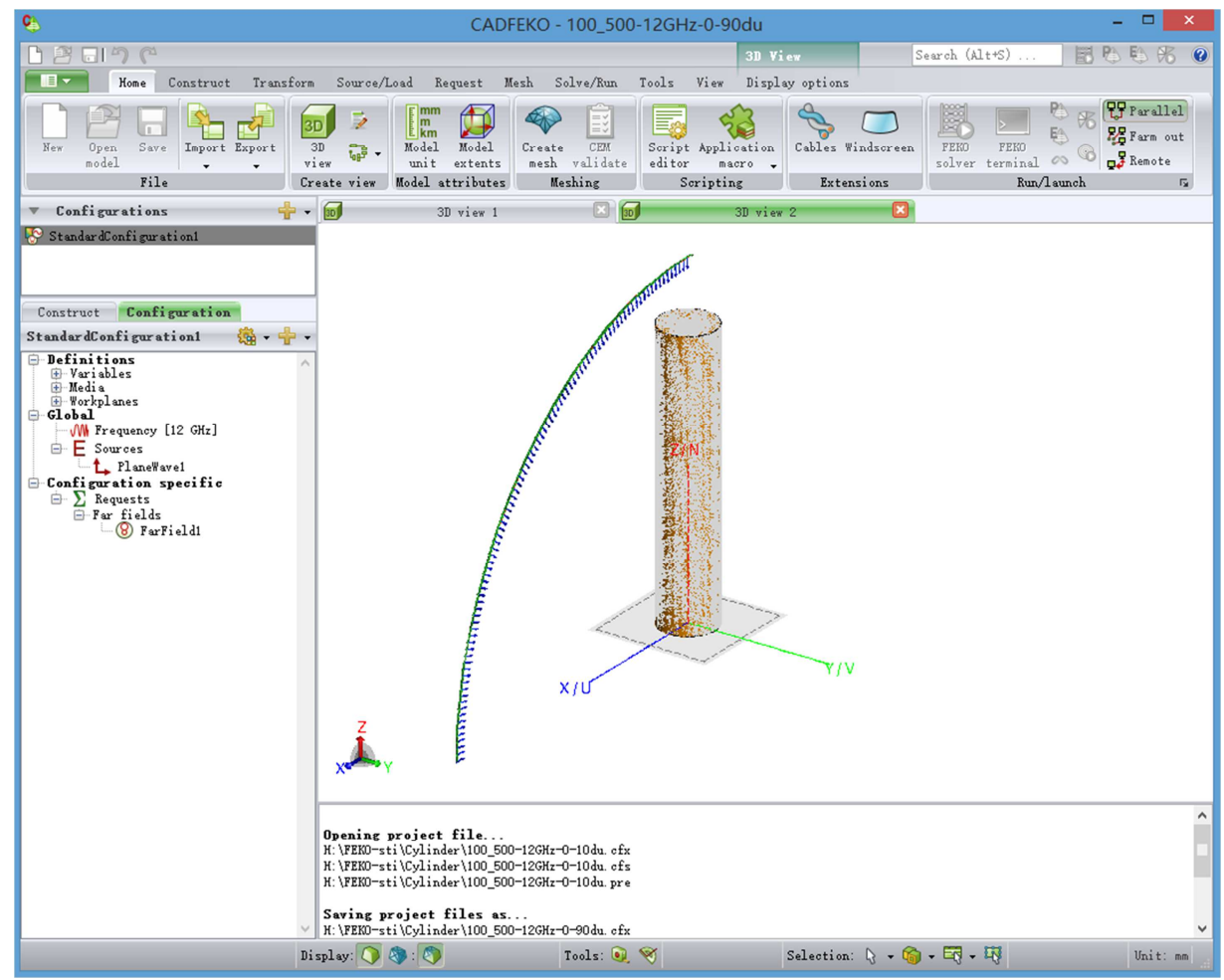

图4 圆柱体RCS计算截图。 


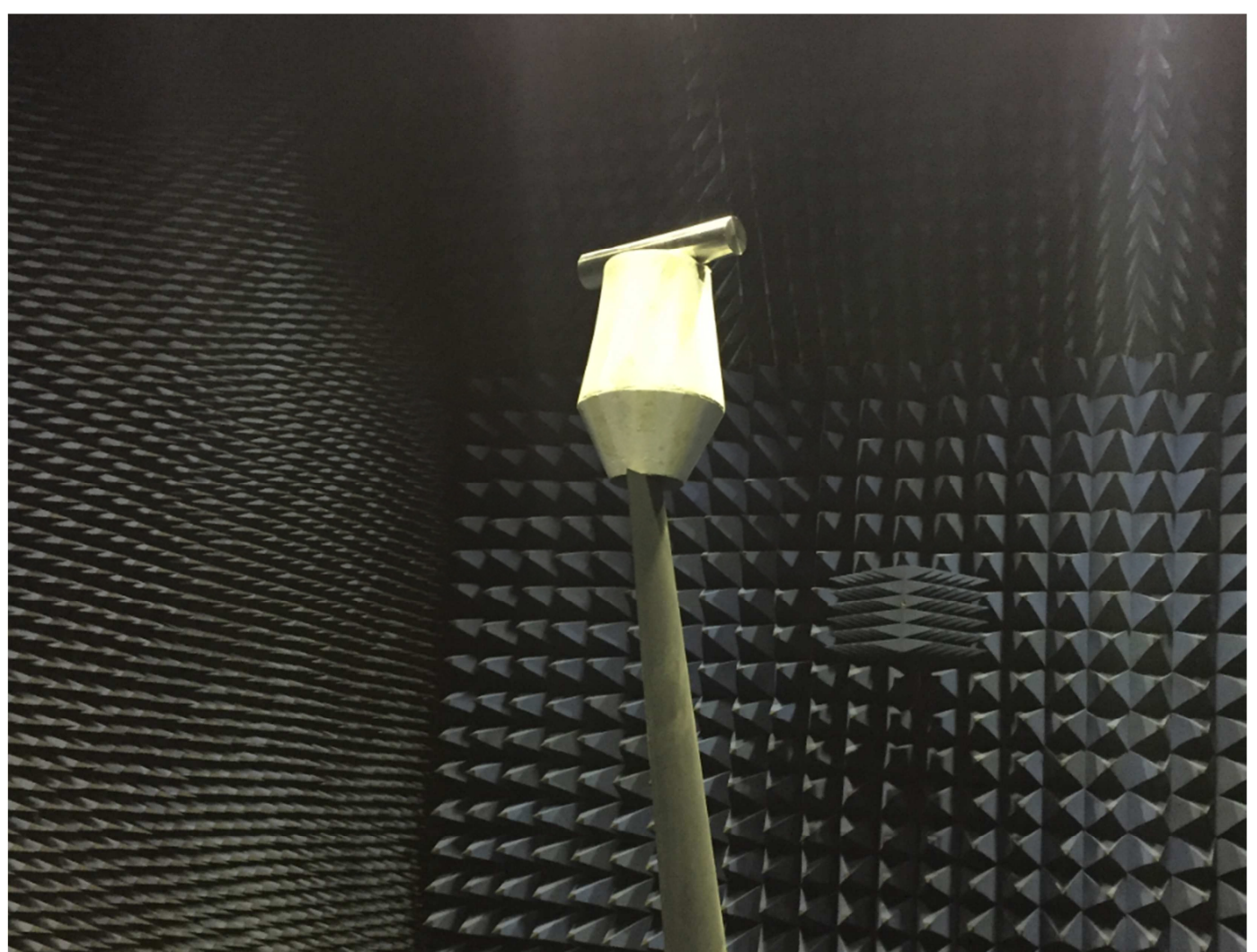

图5 圆柱体RCS测试图片。

表 1 圆柱体 $0^{\circ} \sim 90^{\circ} \mathrm{RCS}$ 计算与测量平均值对比。

\begin{tabular}{lllll}
\hline 圆柱体 $\Phi, \mathrm{L}(\mathrm{mm})$ & 频率f $(\mathrm{GHz})$ & $\sigma_{\mathrm{c}}\left(\mathrm{m}^{2}\right)$ & $\sigma_{\mathrm{m}}\left(\mathrm{m}^{2}\right)$ & $\left|\sigma_{\mathrm{m}}-\sigma_{\mathrm{c}}\right| / \sigma_{\mathrm{m}} \times 100 \%$ \\
\hline 50,100 & 1 & 0.021 & 0.022 & $4.5 \%$ \\
100,100 & 3 & 0.025 & 0.024 & $4.2 \%$ \\
100,500 & 12 & 0.084 & 0.080 & $5.0 \%$ \\
\hline
\end{tabular}

三种圆柱体在相应计算的频率下分别处于瑞利区、谐 振区和光学区, 由图1、2、3及表1可见, 用FEK0 软件计 算的RCS 曲线与测量曲线趋势一致, 在 $0^{\circ} \sim 90^{\circ}$ 范围内直 径 $100 \mathrm{~mm}$ 长度 $500 \mathrm{~mm}$ 圆柱体的 RCS 均值偏差最大, 偏差为 5. $0 \%$, 考虑测量系统的误差因素, 计算结果已经达到了较 高的精度, 可以使用计算值来推测圆柱体的RCS变化情况。

\section{4. 圆柱体RCS计算}

\section{1. 圆柱体 $0^{\circ}$ 入射的RCS计算}

$0^{\circ}$ 入射时圆柱体在入射方向投影为圆，投影面积不 随长度而变化, 圆面边缘产生绕射波, 并在圆柱体表面发 生多次传递和绕射后反射回入射方向对RCS产生影响。由 于圆柱体的对称性, 可分析一种极化的情况, 本文中选用 水平极化。将圆柱体直径设定为 $50 \mathrm{~mm}$, 并选取 $5 \mathrm{~mm} 、 25 \mathrm{~mm}$ 、 $50 \mathrm{~mm} 、 100 \mathrm{~mm} 、 500 \mathrm{~mm} 、 1000 \mathrm{~mm}$ 六个长度的圆柱体在 $1 \mathrm{GHz}$ 、 $3 \mathrm{GHz} 、 6 \mathrm{GHz} 、 9 \mathrm{GHz} 、 12 \mathrm{GHz}$ 频点计算RCS, 计算得到的RCS 曲线如图6所示。

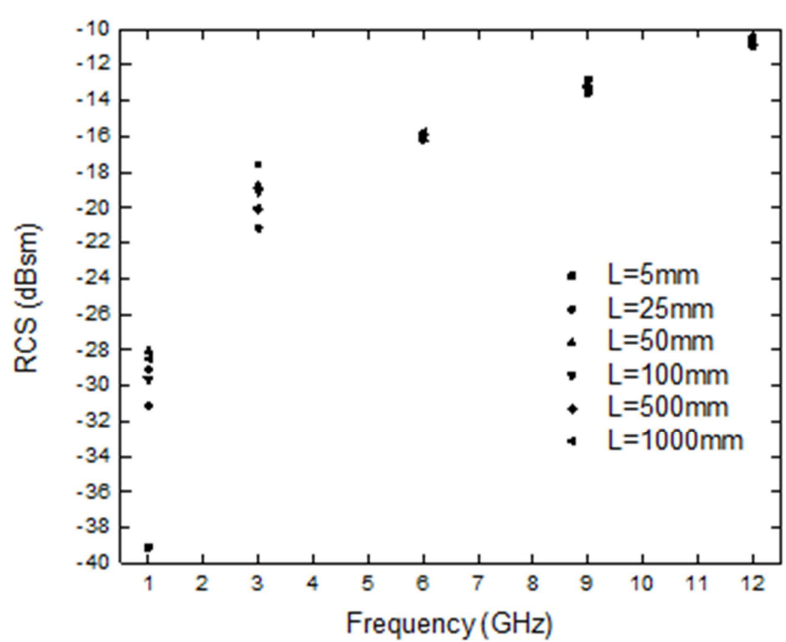

图6 直径 $25 \mathrm{~mm}$ 圆柱体RCS随长度和频率的变化。

在 $1 \mathrm{GHz} 、 3 \mathrm{GHz}$ 不同长度圆柱体 $0^{\circ}$ 入射的 $\mathrm{RCS}$ 差别比较 大, 从 $6 \mathrm{GHz}$ 开始差别变小 RCS 基本一致, 直径对圆柱体的 RCS 影响成为主导作用。由此可见, 当入射波长与圆柱体 
直径相当或小于圆柱体直径时, 不同长度圆柱体 $0^{\circ}$ 入射 的RCS基本稳定波动较小。

\section{2. 圆柱体 $90^{\circ}$ 入射的RCS计算}

$90^{\circ}$ 入射时入射波垂直于柱面，圆柱体在入射方向面 投影为矩形，入射波在柱面阴影区激起的爬行波绕过柱面 会反射回入射方向增强后向RCS [2]。入射波为HH极化时, 电场方向与圆柱体轴线平行, 在圆柱体柱面激起表面行波, 表面行波在圆柱体的柱面与圆面交界处由于金属表面的 不连续引起反射, 进一步影响后向RCS。因此在 $90^{\circ}$ 入射 时将HH极化与VV极化分开计算讨论。

为方便作比较, 选取六组柱面投影面积都为 $10000 \mathrm{~mm}^{2}$ 的圆柱体进行计算 [12], 圆柱体规格如表2所示, 计算频 率选择 $1 \mathrm{GHz} 、 3 \mathrm{GHz} 、 6 \mathrm{GHz} 、 9 \mathrm{GHz} 、 12 \mathrm{GHz}$, RCS计算结果如 图7、8所示。

表2 柱面投影面积为 $10000 \mathrm{~mm}^{2}$ 的圆柱体规格。

\begin{tabular}{llll}
\hline 圆柱体编号 & 直径 $\Phi(\mathrm{mm})$ & 长度 $\mathrm{L}(\mathrm{mm})$ & $\Phi / \mathrm{L}$ \\
\hline $1 \#$ & 10 & 1000 & 0.01 \\
$2 \#$ & 20 & 500 & 0.04 \\
$3 \#$ & 50 & 200 & 0.25 \\
$4 \#$ & 100 & 100 & 1.00 \\
$5 \#$ & 250 & 40 & 6.25 \\
$6 \#$ & 400 & 25 & 16.00 \\
\hline
\end{tabular}

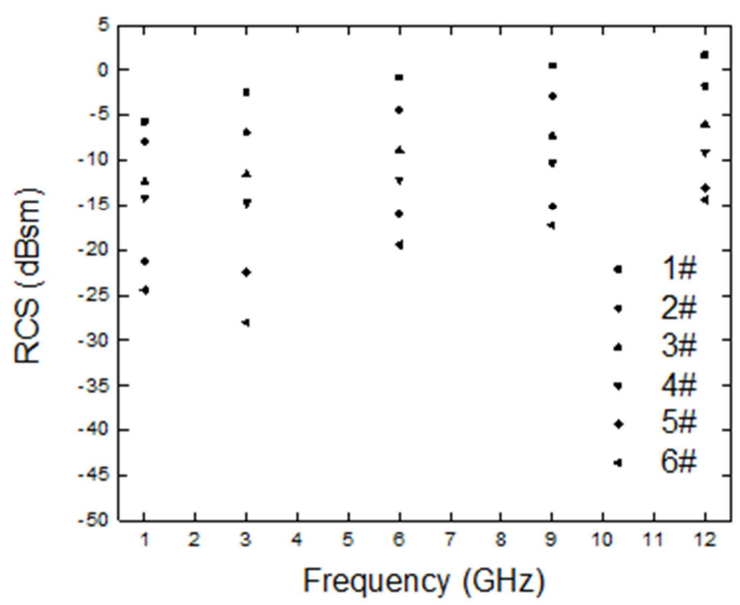

图7 圆柱体 $90^{\circ}$ 入射HH极化的RCS曲线。

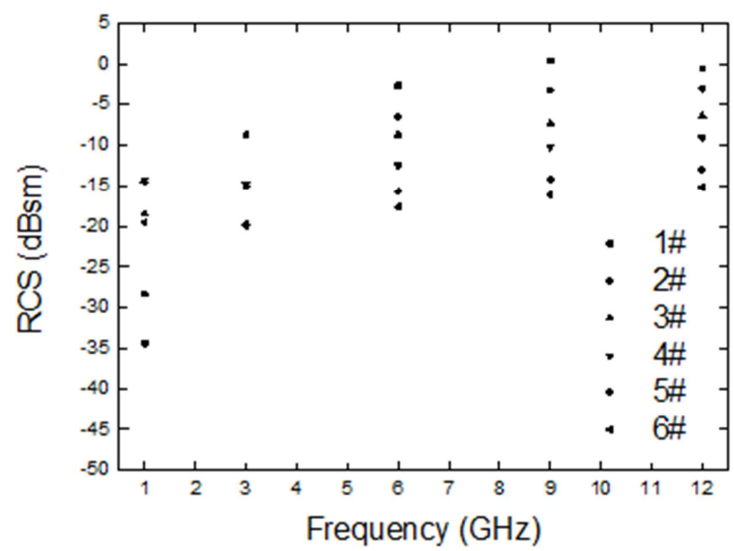

图8 圆柱体 $90^{\circ}$ 入射 $V V$ 极化的RCS曲线。
由表2及图7、8可见，HH极化状态下频率固定时投影 面积为 $10000 \mathrm{~mm}^{2}$ 的圆柱体在 $90^{\circ}$ 入射的RCS随 $\Phi / \mathrm{L}$ 比值增 大而单调减小; VV极化状态下, 在 $1 \mathrm{GHz} 、 3 \mathrm{GHz}$ 时, RCS随 $\Phi / \mathrm{H}$ 比值增大不具有单调减小的特征, 但在 $6 \mathrm{GHz} 、 9 \mathrm{GHz}$ 、 $12 \mathrm{GHz} \operatorname{RCS}$ 随 $\Phi / \mathrm{H}$ 比值增大出现单调减小的特征。

\section{3. 圆柱体掠入射的RCS最大角度计算}

入射角从 $0^{\circ}$ 到 $90^{\circ}$ 变化过程中入射波斜入射到圆面 及柱面, 入射波对圆柱体的作用可分解为 $0^{\circ}$ 与 $90^{\circ}$ 入射 的矢量和, 对于细长光滑结构, 掠入射激起的表面行波最 为重要, 并且只有当入射电场有一个分量与表面相切并在 入射平面内时，才会激励起表面行波 [7]。表面行波方向 图最大值的角度也是掠入射状态下RCS最大的角度, 在工 程应用中使用式（5）来估算表面行波方向图的最大值角 度:

$$
\theta_{e}=49.35 \sqrt{\lambda / l}
$$

式中: $\lambda$ 一入射波波长;

$l$ 一导体长度;

有限长圆柱体在掠入射时圆面反射较强, 而在长度方 向表面行波会产生衰减, 表面行波在柱面与圆面交界处因 导体不连续发生反射又急剧增强后向RCS, 因此有限长圆 柱体在掠入射状态下RCS受到直径和长度的影响因素较多。 计算不同尺寸圆柱体 $0^{\circ}$ 到 $90^{\circ}$ 的RCS, 找出掠入射状态下 最大 RCS 对应的角度 $\theta_{c}$, 并计算该角度与估算值 $\theta_{\mathrm{e}}$ 之间的 偏差可以推断圆柱体尺寸对估算值的影响, 从而有助于合 理使用估算公式判别掠入射时RCS最大的角度。本文中讨 论水平极化的情况, 设计直径 $\Phi$ 为 $1 \mathrm{~mm} 、 5 \mathrm{~mm} 、 10 \mathrm{~mm} 、 20 \mathrm{~mm}$ 长度 $\mathrm{L}$ 为 $25 \mathrm{~mm} 、 50 \mathrm{~mm} 、 100 \mathrm{~mm} 、 250 \mathrm{~mm} 、 500 \mathrm{~mm}$ 共 20 种规格的 圆柱体, 选择 $6 \mathrm{GHz}$ 计算 $\theta_{\mathrm{c}}$, 并按照式 (6) 计算 $\theta_{\mathrm{c}}$ 与 $\theta_{\mathrm{e}}$ 的偏差比Deviation（D）:

$$
\mathrm{D}=\left|\theta_{\mathrm{c}}-\theta_{\mathrm{e}}\right| / \theta_{\mathrm{c}} \times 100 \%
$$

将偏差比D与圆柱体尺寸做成曲线如图9所示。

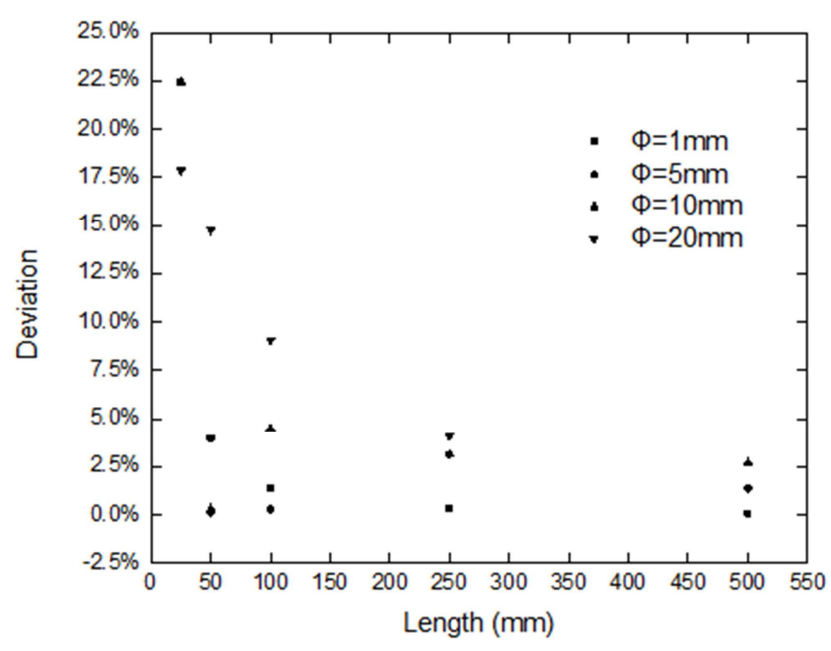

图9 掠入射RCS最大值角度的计算与估算偏差比。 
由图9可见, 圆柱体长度为 $25 \mathrm{~mm}$ 时, $l / \lambda=0.5,4$ 种直 径圆柱体的偏差比均超过 $17.5 \%$; 圆柱体长度为 $50 \mathrm{~mm}$ 时, $l$ $/ \lambda=1$, 直径为 $1 \mathrm{~mm} 、 5 \mathrm{~mm} 、 10 \mathrm{~mm}$ 的圆柱体偏差比小于 $5.0 \%$, 而直径为 $20 \mathrm{~mm}$ 的圆柱体偏差比为 $14.7 \%$; 圆柱体长度为 $100 \mathrm{~mm}$ 时, $l / \lambda=2$, 直径为 $1 \mathrm{~mm} 、 5 \mathrm{~mm} 、 10 \mathrm{~mm}$ 的圆柱体偏差 比小于 $5.0 \%$, 而直径为 $20 \mathrm{~mm}$ 的圆柱体偏差比为 $9.1 \%$; 圆柱 体长度为 $250 \mathrm{~mm}$ 和 $500 \mathrm{~mm}$ 时, $l / \lambda \geqslant 5,4$ 种直径的圆柱体偏 差比均小于 $5.0 \%$ 。通过分析可推测:

(1) 圆柱体长度小于入射波长时，圆柱体直径对偏 差影响较小, 使用估算公式计算的最大角度与准确计算结 果均相差很大;

（2）圆柱体长度与入射波长尺寸相当时，直径对偏 差影响明显, 直径远小于入射波长的情况下估算公式仍可 得到较准确的最大值角度;

(3) 圆柱体长度远大于入射波长时，圆柱体直径对 偏差影响很小, 直径小于入射波长的情况下均可得到很小 的偏差比，可以使用估算公式来计算最大值角度。

\section{5. 结束语}

（1）通过计算与测量分析，使用FEKO软件计算圆柱 体的RCS均具有很高的精度。

（2）入射波垂直于圆面时，圆柱体直径对RCS影响明 显, 圆柱体长度对RCS影响较小; 入射波长与圆柱体直径 相当或小于圆柱体直径时, RCS随长度变化很小。

(3) 入射波垂直于柱面时, 极化方式对RCS影响明显, 水平极化条件下相同投影面积的圆柱体RCS随直径与长度 的比值增大而单调减小; 垂直极化时圆柱体RCS 随直径与 长度的比值增大不具有单调减小的特征, 但随频率的升高 出现单调减小的特征。

（4）表面行波方向图最大值角度在圆柱体长度远大 于直径和入射波长时使用估算公式可获得较准确的结果; 在圆柱体长度小于入射波长时估算结果偏差很大, 不能 使用; 在圆柱体长度与入射波长尺寸相当时直径对估算 结果影响很大, 直径远小于入射波长时仍可使用估算公 式。

本文中总结的圆柱体RCS变化规律是基于离散尺寸 的圆柱体在离散频率点的RCS计算, 在应用中对于入射 波长与圆柱体直径或长度相当的情况, 具体的RCS波动 或偏差需要进一步计算确定。圆柱体的直径、长度与入 射波长的比值以及入射角度范围需要细化分类, 对 RCS 的详细影响需要进一步研究探讨, 这也是未来研究工作 的方向。

\section{致谢}

本文为北京航空材料研究院创新基金项目《典型目标 体的RCS减缩规律研究》 (编号: KJSJ140912) 的阶段性成 果之一。

\section{参考文献}

[1] 李有权, 胡林华, 杨志荣. TE波照射下圆柱RCS计算方法的比 较 $[J]$. 空军雷达学院学报, 2005, 19（3）：28-30。

[2] 郝雷, 张维生, 王天民, 武哲. 理想导电圆柱爬行波后向RCS 的计算与测量研究. 国防科学: 中国科协第四届优秀博士生 学术年会论文集 $[C] .2006$ ：1569-1574。

[3] 夏学仁, 黄泽贵, 邓发升. GTD-MOM 技术求解细理想导电圆 柱电磁散射 $[J]$. 空军工程大学学报, 2002, 3 (5) : 72-75。

[4] 郭华昌, 侯新宇, 张晓金, 张玉英. 应用SBR / FDTD混合算 法求解圆柱形复杂终端腔体RCS [J]. 电子测量技术, 2007, 30 (8) : 203-206。

[5] 徐仁香. 基于高频方法的RCS建模及应用 [D]. 南京: 南京理 工大学, 2013:6。

[6] 李小华.复杂目标雷达散射截面频率特性研究 [D]. 成都: 电 子科技大学, 2007: 3 。

[7] 刘战合, 黄沛霖, 武哲. 飞行器目标频率响应散射特性 [J]. 航空学报，2009，30（4）：643-648。

[8］杨端义. 复杂目标雷达散射截面的分析方法 [D]. 南京: 南京 理工大学, 2009:2。

[9] E. F. 克拉特. 雷达散射截面-预估、测量和减缩 [M]. 阮颖铮, 陈海, 译. 北京: 电子工业出版社, 1988:78-81。

[10］郭鹏, 白亮, 武梦洁, 蒋宏. 基于FEK0的雷达散射截面实时 计算 $[\mathrm{J}]$.航空科学技术: 2013 , (6) : 72-76。

[11] Shinichiro Ohnuki, Noriaki Ohtaka, Takashi Hinata. Electromagnetic Scattering from Rectangular Cylinders with Wedge Cavities. IEEE Antennas \& Propagation Society International Symposium[C]. 2006: 2925-2928.

[12] Yong Li, Wenzhu Li, Li Yuan, Linxing Yang . Research on RCS characteristic of three kinds of metal plate. 2012 IEEE 2nd International Conference on Lloud Computing and Intelligence Systems [C]. 2012: 875-878. 